\title{
Metallicities at the Sites of Nearby SN and Implications for the SN-GRB Connection
}

\author{
Maryam Modjaz $^{1,2}$ L. Kewley ${ }^{3}$, R. P. Kirshner ${ }^{2}$, K. Z. Stanek ${ }^{4}$, \\ P. Challis ${ }^{2}$, P. M. Garnavich ${ }^{5}$, J. E. Greene ${ }^{6}$, P. L. Kelly ${ }^{7}$, \& \\ J. L. Prieto ${ }^{4}$ \\ ${ }^{1}$ UC Berkeley Astronomy Department \\ email: mmodjaz@astro.berkeley.edu \\ ${ }^{2}$ Harvard-Smithsonian CfA \\ email: kirshner,pchallis@cfa.harvard.edu \\ ${ }^{3}$ Department of Astronomy, Univerisity of Hawaii \\ email: kewley@IfA. Hawaii.Edu \\ ${ }^{4}$ Department of Astronomy, The Ohio State University \\ email: kstanek,prieto@astronomy.ohio-state.edu \\ ${ }^{5}$ Department of Physics, University of Notre Dame \\ email: pgarnavi@nd.edu \\ ${ }^{6}$ Department of Astrophysical Sciences, Princeton University \\ email: jgreene@astro.princeton.edu \\ ${ }^{7}$ Kavli Institute for Particle Astrophysics and Cosmology \\ email: pkelly3estanford.edu
}

\begin{abstract}
While the broad-lined Type Ic supernovae (SN Ic-bl) associated with long-duration gamma-ray bursts (GRBs) have been studied, we do not fully understand the conditions that lead to each kind of explosion in a massive star. Here we show clues as to the production mechanism of GRBs by comparing the chemical abundances at the sites of 5 nearby $(z<0.25)$ broad-lined SN Ic that accompany nearby GRBs with those of 12 nearby $(z<0.14)$ broad-lined SN Ic that have no observed GRBs. We show that the oxygen abundances at the GRB sites are systematically lower than those found near ordinary broad-lined SN Ic. A unique feature of this analysis is that we present new spectra of the host galaxies and analyze the measurements of both samples in the same set of ways, using 3 independent metallicity diagnostics. We demonstrate that neither SN selection effects (SN found via targeted vs. non-targeted surveys) nor the choice of strong-line metallicity diagnostic can cause the observed trend. Though our sample size is small, the observations are consistent with the hypothesis that low metal abundance is the cause of some massive stars becoming SN-GRB. We derive a cut-off metallicity of $0.2-0.6 \mathrm{Z}_{\odot}$, with the exact value depending on the adopted metallicity scale and solar abundance value.
\end{abstract}

Keywords. gamma rays: bursts- supernovae: individual - galaxies: abundances

\section{Introduction}

We seek clues as to the production mechanism of long-durations gamma-ray bursts (GRBs) by comparing the chemical abundances at the sites of nearby broad-lined SN Ic (SN Ic) that accompany some GRBs with the broad-lined SN Ic that have no observed GRBs. In nearby $(z<0.25)$ long-duration GRBs, after the afterglow has faded, if the spectrum of the underlying event is observed, it is that of a broad-lined SN Ic (Galama et al. 1998, Stanek et al. 2003, Hjorth et al. 2003, Modjaz et al. 2006). These are SN Ic whose optical spectra show no hydrogen or helium lines and whose line widths approach $30,000 \mathrm{~km} \mathrm{~s}^{-1}$ (2-3 times larger than normal SN Ic, see Figure 1), also known as 


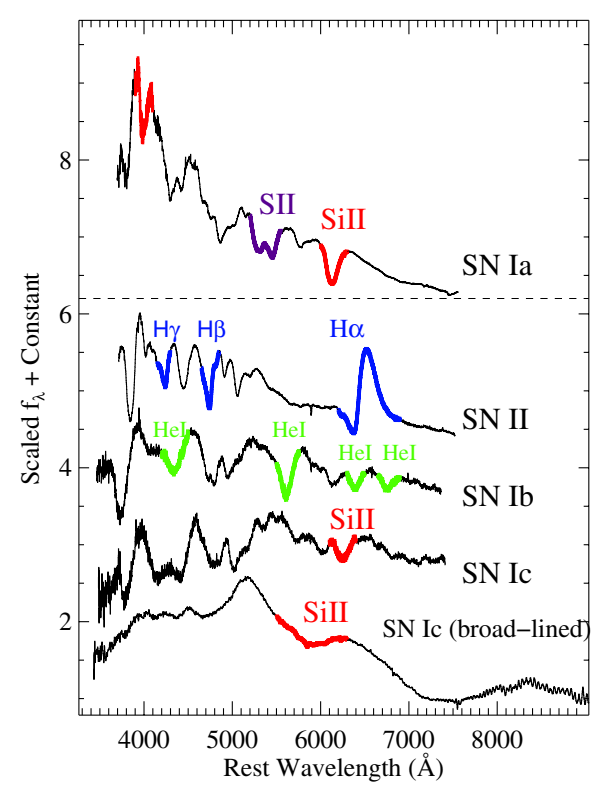

Figure 1. Representative sample of SN spectra illustrating the main SN classifications (but not all of them), which are based on the presence or absence of certain elements in the SN spectrum at maximum light. The main line identifications are marked. Broad-lined SN Ic (bottom spectrum) are the SN spectroscopically identified in the afterglow of nearby long GRBs. The SN shown are: SN 1994D (SN Ia; Meikle et al. 1996); SN 1999em (SN II; Leonard et al. 2002), SN 2004gq (SN Ib; Modjaz 2007), SN 2004gk (SN Ic; Modjaz 2007), and SN 1998bw (broad-lined SN Ic; Galama et al. 1998).

"Hypernovae". However, a growing number of broad-lined SN Ic are also seen without accompanying GRBs. If low metallicity is necessary for producing conditions suitable for GRBs, as proposed by observational studies (e.g., Stanek et al. 2006) and theoretical work (e.g., Yoon \& Langer 2005, Woosely \& Heger 2006), then we would expect broadlined SN Ic intrinsically without GRBs to erupt in higher metallicity environments. This is the hypothesis we test here, using the best available list of SN and our own uniform determinations of the local metal abundance. Details of this work are presented in Modjaz et al. (2008a).

\section{Sample and Observations}

\subsection{SN Host Galaxy Samples}

For our sample of SN connected with GRBs, we considered the four nearby secure cases of direct, and spectroscopically determined, SN-GRB associations, namely GRB 980425 / SN 1998bw, GRB 030329/SN 2003dh, GRB 031203/SN 2003lw and GRB/XRF 060218 / SN 2006aj. We also include the host of XRF 020903 that had a clear supernova signature in its light curve and in its afterglow spectrum, but we do note that this SN confirmation has a lower degree of certainty than the other GRB-SN associations. In order to derive oxygen abundances, we used the published emission-line fluxes for the sites of the SNGRBs, when possible, and otherwise the light-integrated host galaxy spectra (see Modjaz et al. $2008 \mathrm{~b}$ for a full list of references).

We obtained and analyzed host galaxy spectra of a total of 12 broad-lined SN Ic without observed GRBs. A few of these broad-lined SN Ic are well-documented in the literature, 
and to increase our sample, we searched for additional ones in the announcements of the International Astronomical Union Circulars. Half of our sample consists of host galaxies of SN that were found via traditional galaxy-targeted SN surveys (e.g., Lick Observatory SN Search), whereas the other half consists of galaxies in which the SN were found via large field-of-view or rolling surveys, i.e., where the host galaxy was not targeted (e.g., Texas SN survey, SDSS-II SN survey). We are aware that selection effects can be introduced by different methods of discovery. To mitigate these selection effects, we worked hard to include SN that were found in host galaxies that had not been targeted for search. We believe they provide a better match to the host galaxies that are selected by the appearance of a GRB. For completeness, we analyze and discuss spectra of all broad-lined SN Ic. But when directly comparing to the sample of SN Ic connected with GRBs, we identify the SN Ic found in the same, non-targeted fashion in order to minimize discovery selection effects. The sample size of broad-lined SN Ic that are free of galaxyselection effects is comparable to that of SN-GRB. More details about the sample are given in Modjaz et al. (2008a).

\subsection{Observations and Metallicity Measurements}

We obtained spectra of the SN sites and of their galaxy nuclei via our own observations (with the $6.5 \mathrm{~m}$ Magellan, the $6.5 \mathrm{~m}$ MMT, and 1.5m Mt. Hopkins telescopes) and archival data (SDSS DR5). Using the galaxy emission-line measurements corrected for stellar absorption and extinction, we derive central oxygen abundances and abundances at the SN position, while explicitly including the statistical uncertainties in the line measurements. We compute oxygen abundances via strong-line diagnostics using three independent and well-known calibrations: Kewley \& Dopita (2002), McGaugh (1991), and Pettini \& Pagel (2004), the latter being effectively in the direct electron temperature $\left(T_{e}\right)$ scale. Furthermore, we compute local star formation rates, and draw from the literature the host galaxy B-band luminosities, $M_{B}$. We compare the properties of our sample with properties of the 5 nearby SN-GRB hosts for which we derive chemical abundances using published emission line fluxes via the same metallicity diagnostics as for the SN without GRBs in our sample. Furthermore, we include the sample of local $(0.005<z<0.2)$ SDSS star forming galaxies (Tremonti et al. 2004), whose abundances we computed in the same three scales using their published line fluxes.

\section{Discussion}

Figure 2 shows the main result of our comparison: we plot host galaxy metallicity (as expressed in terms of oxygen abundance $12+\log (\mathrm{O} / \mathrm{H})$ with the KD02 calibration) and host galaxy luminosity $\left(M_{B}\right)$ of broad-lined SN Ic without observed GRBs (called "SN Ic (broad)", circles) and with GRBs (called "SN Ic (broad \& GRB)", squares). Objects whose host galaxies had not been targeted during the discovery have an extra circle (for SN without GRBs) or an extra square (for SN with GRBs) around their plotted symbol. We note that three of the five broad-lined SN Ic found in the lower luminosity galaxies $\left(M_{B}>-19 \mathrm{mag}\right)$ were discovered by the SDSS-II SN Survey, which is a galaxy-impartial survey. For the broad-lined SN Ic we only plot abundances measured at or extrapolated to the SN position. These plotted values are lower than the values we measure for the center of the same host galaxies presumably due to metallicity gradients. The SN-GRB host abundances also reflect the metallicity at the SN-GRB position, since they were either measured specifically at the SN position (for GRB 980425/SN 1998bw), or the SN reside in the nucleus of their dwarf-galaxy hosts that are chemically homogeneous (Kobulnicky et al. 1997). Due to the short life times of the massive SN progenitor stars 


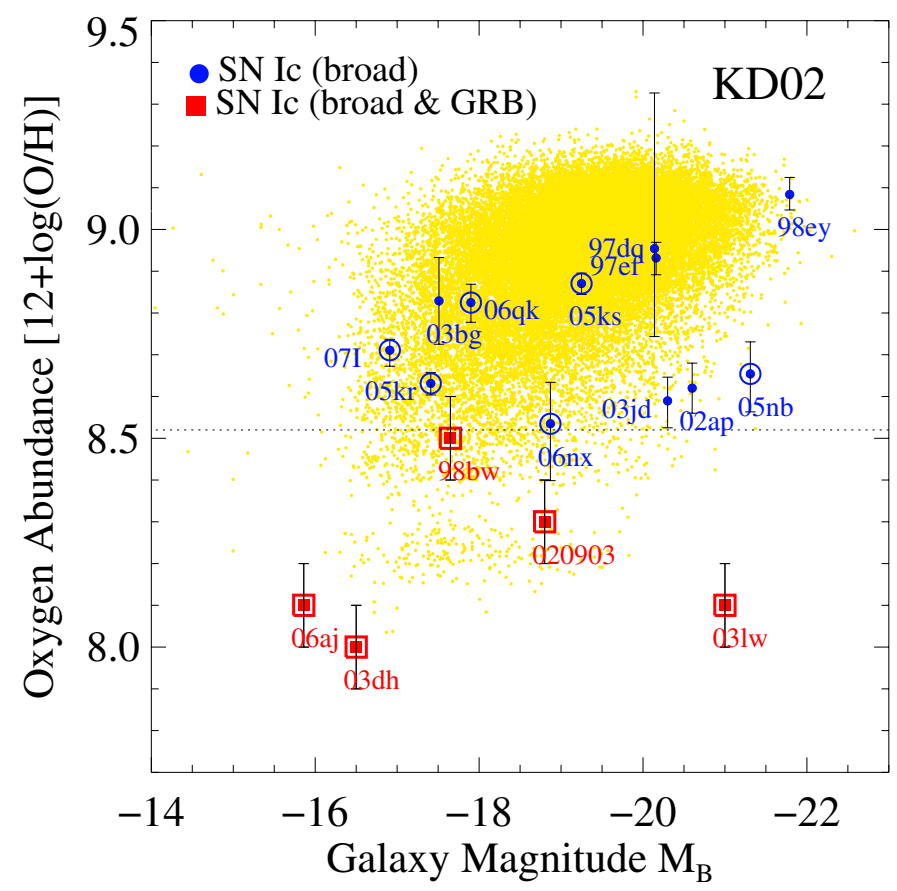

Figure 2. Host galaxy luminosity $\left(M_{B}\right)$ and host galaxy metallicity (in terms of oxygen abundance) at the sites of nearby broad-lined SN Ic ("SN Ic (broad)": filled circles) and broadlined SN Ic connected with GRBs ("SN (broad \& GRB)": filled squares; see also Stanek et al. (2006), Kewley et al. (2007)). Extra circles and squares designate SN which were found in a non-targeted fashion. The oxygen abundances are in the Kewley \& Dopita (2002) (KD02) scale and represent the abundance at the SN position. Due to radial metallicity gradients, the gas abundance at the SN position is lower than the central galaxy abundance for some SN. Labels represent the SN names while one ("020903") refers to its associated GRB. Yellow points are values for local star-forming galaxies in SDSS Tremonti et al. (2004), re-calculated in the Kewley \& Dopita (2002) scale for consistency, and illustrate the empirical luminosity-metallicity $(L-Z)$ relationship for galaxies. Host environments of GRBs are systematically less metal-rich than host environments of broad-lined SN Ic where no GRB was observed, even for the same range of host luminosities. The dotted line at $12+\log (\mathrm{O} / \mathrm{H})_{\mathrm{KD} 02} \sim 8.5$ designates the apparent dividing line between SN with and without observed GRBs.

(<10 Million years for $M_{\mathrm{ZAMS}}>20 \mathrm{M}_{\odot}$ ), we regard the metallicities at the SN position as natal metallicities.

Moreover, we plot in Figure 3 the comparison between the two host samples and the local SDSS galaxies in the metallicity scales of M91 and of PP04-O3N2, with the latter being effectively in the $T_{e}$ scale. While the absolute values of the abundances are different in different scales, as expected, the bimodal distribution persists in each scale and thus, is independent of the choice of metallicity diagnostic. The K-S Test applied to the host abundances of SN Ic (broad \& GRB) and SN Ic (broad) found in a nontargeted fashion yields low probabilities of $4 \%$ (M91-based abundances) and $3 \%\left(T_{e}\right.$ based abundances) that they are drawn from the same population. For each scale, we plot as a dotted line the boundary that separates the two samples: $12+\log (\mathrm{O} / \mathrm{H})_{\mathrm{M} 91} \sim 8.4$ and $12+\log (\mathrm{O} / \mathrm{H})_{T_{e}} \sim 8.1$. Although our sample is small our findings are consistent with the hypothesis that low metal abundance is the cause of some very massive stars 


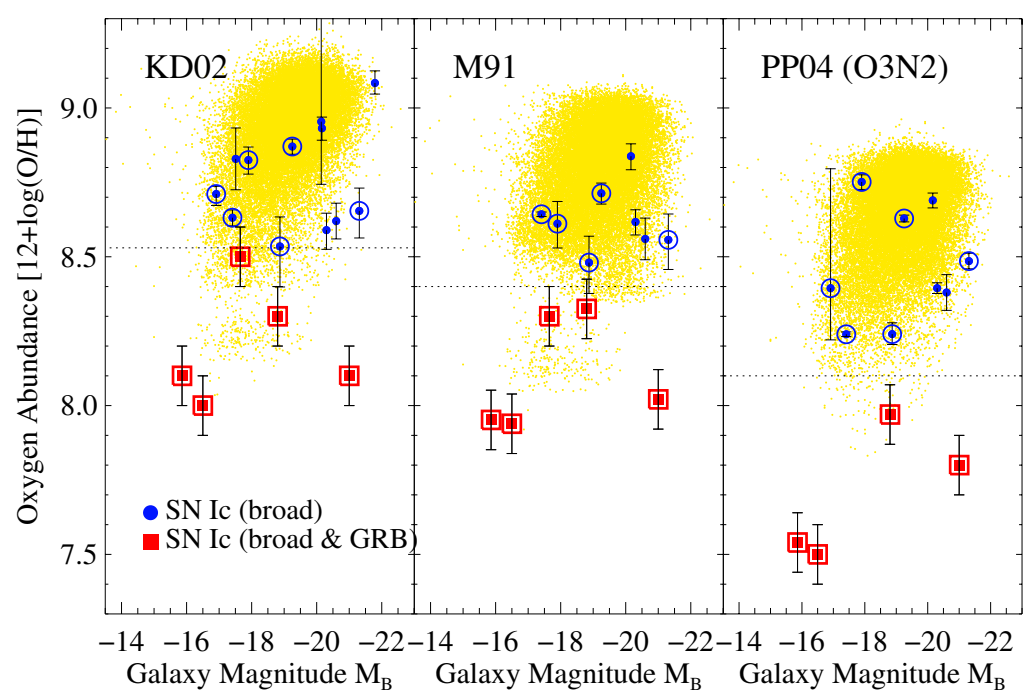

Figure 3. Similar to Figure 2, but using different metallicity diagnostics; Kewley \& Dopita (2002) (KD02) as in Figure 2 (left); McGaugh (1991) (M91, middle); and Pettini \& Pagel (2004) (PP04-O3N2), which is effectively on the $T_{e}$ scale, (right). Host environments of GRBs are more metal-poor than environments of broad-lined SN Ic where no GRB was observed, independent of the abundance scale used. Yellow dots designate the SDSS galaxy values calculated in the respective metallicity scales.

becoming SN-GRB. The absolute value for the cut-off metallicity ranges between $0.2-0.6$ $\mathrm{Z}_{\odot}$ depending on the adopted metallicity scale and solar abundance value.

At face value, our results differ from various studies in the literature, which have concluded that high- $z$ GRB hosts are not necessarily low-metallicity systems. However, there might be intrinsic differences in GRB population at low and high $z$ (e.g., Guetta \& Della Valle 2006): the low- $z$ GRBs might constitute a different class of low-luminosity GRBs that are not detected at higher $z$. Moreover, most of the techniques for measuring abundances at higher $z$ are different from our direct approach, as they use absorption techniques in GRB afterglows (Prochaska et al. 2007 and references therein).

Radio observations do not support off-axis GRBs with broad-lined SN Ic in half of our sample (Soderberg et al. 2006), while we cannot exclude the possibility of off-axis GRBs in the rest of the SN sample. Recently, Mazzali et al. (2005) argued for an off-axis jet in one SN of our sample, SN 2003jd (see Figure 2), since they interpreted double-peaked oxygen profiles observed in its late-time spectra as signs of an aspherical, axisymmetric explosion caused by a jet. However, a large set of SN with nebular spectra exhibit doublepeaked oxygen profiles, which indicates that asphericities are common in normal SN Ib/c (Modjaz et al. 2008b, Maeda et al. 2008) and not necessarily a sign of an off-axis GRB.

\section{Conclusions}

We presented spectroscopic data of a statistically significant set of host galaxies of 12 nearby $(z<0.14)$ broad-lined SN Ic with no observed GRBs. Using the galaxy emissionline measurements corrected for stellar absorption and extinction, we derived central oxygen abundances and abundances at the SN position based on strong-line diagnostics in three independent scales. We compared the properties of our host sample with the 
properties of five nearby SN-GRB hosts, for which we derived chemical abundances using the same three metallicity diagnostics as for SN without observed GRBs. Broad-lined SN Ic without GRBs tend to consistently inhabit more metal-rich environments, and their host galaxies, for the same luminosity range $\left(-17<M_{B}<-21 \mathrm{mag}\right)$, are systematically more metal-rich than corresponding GRB host galaxies. The trend is independent of the choice of diagnostic and cannot be due to selection effects as we include six SN found in a similar non-targeted manner as GRB-SN.The boundary between broad-lined SN Ic that have a GRB accompanying them and broad-lined SN Ic without a GRB lies at an oxygen abundance of $\sim 12+\log (\mathrm{O} / \mathrm{H})_{\mathrm{KD} 02} \sim 8.5$, which corresponds to $0.2-0.6 \mathrm{Z}_{\odot}$ depending on the adopted metallicity scale and solar abundance value.

M.M. acknowledges support from the Miller Foundation for the time during which part of this study was conducted and thanks the organizers of IAUS 250 for an enjoyable and enlightening conference. Supernova research at Harvard University has been supported in part by the National Science Foundation grant AST06-06772.

\section{References}

Galama, T. J., Vreeswijk, P. M., van Paradijs, J. et al. 1998, Nat, 395, 670

Guetta, D. \& Della Valle, M. 2006, ApJ, 657, L73

Hjorth, J., Sollerman, J., Møller, P. et al. 2003, Nat, 423, 847

Kewley, L. J. \& Dopita, M. A. 2002, ApJS, 142, 35

Kewley, L. J., Brown, W. R., Geller, M. J. et al. 2007, AJ, 133, 882

Leonard, D. C., Filippenko, A. V., Gates, E. L. et al. 2002, PASP, 114, 35

McGaugh, S. S. 1991, ApJ, 380, 140

Maeda, K., Kawabata, K., Mazzali, P. A. et al. 2008, Sci, 319, 1220

Meikle, W. P. S., Cummings, R. J., Geballe, T. R. et al. 1996, MNRAS, 281, 263

Modjaz, M., Stanek, K. Z., Garnavich, P. M. et al. 2006, ApJ, 645, L21

Modjaz, M. 2007, Ph. D. Thesis, Harvard University

Modjaz, M., Kewley, L., Kurshner, R. P. et al. 2008a, AJ, 135, 1136

Modjaz, M., Kirshner, R. P., \& Challis, P. 2008b, ApJL, submitted (arXiv:0801.0221)

Pettini, M. \& Pagel, B. E. J. 2004, MNRAS, 348, L59

Soderberg, A. M., Nakar, E., Berger, E., \& Kulkarni, S. R. 2006, ApJ, 638, 930

Stanek, K. Z., Matheson, T., Garnavisch, P. M. et al. 2003, ApJ 591, L17

Stanek, K. Z., Gnedin, O. Y., Beacom, J. F. et al. 2006, Acta Astronomica, 56, 333

Tremonti, C. A., Heckman, T. M., Kauffmann, G. et al. 2004, ApJ, 613, 898

Woosley, S. E. \& Heger, A. 2006, ApJ, 637, 914

Yoon, S.-C. \& Langer, N. 2005, A\&A, 443, 643

\section{Discussion}

HIRSCHI: Very interesting! Where would the LMC and SMC oxygen abundances lie on your plots?

Modjaz: The values for the $\mathrm{LMC}$ and the $\mathrm{SMC}$ are $\log (\mathrm{O} / \mathrm{H})+12=8.3$ and 8.0 in the Kewley \& Dopita scale, respectively. So exactly where the GRB-SN Hosts lie.

MACFADYEN: So there must be something fundamentally important about the impact of low metallicity on the GRB progenitors?

ModJAz: Yes. I hope I have convinced you that, observationally, we tried to be very thorough by taking selection effects and metallicity systematics into account, and this is what the data are telling us. 